\section{Re-evaluación de seroprevalencia de virus hepatitis $E$}

\section{Natalia Covarrubias, Carmen Hurtado, Alex Díaz, Gabriel Mezzano, Javier Brahm y Mauricio Venegas}

\section{Hepatitis E virus seroprevalence: a reappraisal}

Reported seroprevalence of hepatitis E virus (HEV) in developed countries is between $0.3-53 \%$. Published data relies on the assays used and its technical performance. Sensitivity on new available tests has improved, which has changed HEV seroprevalence around the world. We re-evaluated retrospectively, 178 serum samples of patients with previous anti HEV IgG determination between 2009 and 2012. Initial analysis was performed with ELISA kit Genelabs (Singapore), with 7.3\% positivity. The reevaluation was done with ELISA kit AccuDiag ${ }^{\mathrm{TM}} \mathrm{HEV}-$ IgG (Diagnostic Automation, United States), with reported sensitivity and specificity over $99.8 \%$. With the new assay, $32.6 \%$ positive samples were found, significantly greater to the previous result $(p<0.001)$ (4.5 times more). There were no differences in gender but a significant association between age and HEV IgG seropositivity was found ( $p<$ 0.001 ). This suggests that previous testing might have underestimated HEV seroprevalence in Chile, which should be reevaluated using the new available test.

Key words: Hepatitis E Virus, seroprevalence, IgG antibody.

Palabras clave: Virus hepatitis E, seroprevalencia, anticuerpos IgG.

\section{Introducción}

E 1 virus hepatitis $\mathrm{E}$ (VHE) es un virus ARN pequeño, sin envoltura, único miembro de la familia Hepeviridae. Se han identificado cuatro genotipos que pueden infectar a mamíferos, con distinta distribución geográfica. La infección por VHE es considerada una enfermedad emergente de importancia global, siendo una de las principales causas de hepatitis de transmisión entérica ${ }^{1}$.

El curso de la infección por VHE es generalmente benigno. Sin embargo, se ha observado la asociación con hepatitis fulminante grave en mujeres embarazadas y en pacientes con enfermedad hepática subyacente. La infección crónica por el VHE genotipo 3 ha sido descrita en individuos inmunocomprometidos, como en receptores de trasplante $\mathrm{y}$ en pacientes sometidos a quimioterapia, los cuales progresan rápidamente a una enfermedad hepática avanzada².

Hospital Clínico Universidad de Chile, Santiago, Chile.

Departamento de Medicina, Sección de Gastroenterología.

Conflictos de interés: no existen.

Fuente de Financiamiento: Oficina de Apoyo a la Investigación Clínica del Hospital Clínico Universidad de Chile.

Recibido: 12 de diciembre de 2014 / Aceptado: 3 de junio de 2015

Correspondencia a:

Natalia Covarrubias

ncovarrubias@hcuch.cl
Se estima que en el mundo alrededor de 2,3 billones de personas han tenido contacto con el VHE. Se han descrito como zonas endémicas gran parte de Asia, África, la región del Mediterráneo, México y América del Sur ${ }^{1,3}$. En países europeos se reportan seroprevalencias de VHE entre 0,3 y $53 \%$. Esta alta variabilidad es probablemente atribuible a las condiciones sanitarias y los hábitos alimenticios de la población estudiada. Sin embargo, la sensibilidad de los ensayos de anticuerpos del tipo IgG anti-VHE utilizados también puede influir en los resultados y, posiblemente, sea la causa más importante de estas diferencias. ${ }^{4}$.

En Latinoamérica, se describe una endemicidad moderada. La mayoría de los estudios muestran tasas de prevalencia en población rural y urbana que varían entre $1 \%$ y $10 \%$, con evidentes diferencias entre las distintas regiones y cifras bastante superiores en las comunidades indígenas, que alcanzan valores tan altos como $17 \%$ en población mapuche de Chile y hasta $41 \%$ en población indígena amazónica de Bolivia². En Chile, existe escasa información al respecto, destacando dos estudios previos realizados en donantes de sangre en los años 1996 y 1997 con seroprevalencias de 4\% en Santiago y 8\% en Valdivia, Osorno y Puerto Montt, respectivamente ${ }^{5,6}$.

Se han desarrollado múltiples ensayos para la detección de anticuerpos IgG anti-VHE disponibles para su uso, aunque ninguno de ellos ha sido aprobado por la Administración de Drogas y Alimentos de E.U.A (FDA). Diversos estudios muestran resultados muy variables entre los distintos ensayos, lo cual podría explicar las diferentes estimaciones de seroprevalencia de VHE en algunas poblaciones. El uso de pruebas más sensibles de IgG anti-VHE ha llevado a aumentar de tres a cuatro veces las estimaciones de seroprevalencia ${ }^{7}$.

El objetivo de esta investigación, fue reevaluar la seroprevalencia de VHE utilizando un nuevo ensayo disponible.

\section{Material y Método}

Se estudiaron en forma retrospectiva, 178 muestras de suero de pacientes con análisis previo de anticuerpos IgG anti-VHE en el Laboratorio de Gastroenterología del Hospital Clínico de la Universidad de Chile, entre los años 2009 y 2012. Las muestras se encontraban almacenadas a $-20{ }^{\circ} \mathrm{C}$. El uso de las muestras fue aprobado por el comité de ética local.

El análisis previo de las muestras se había realizado con la prueba ELISA Genelabs (Singapur), donde 7,3\% resultaron positivas para IgG anti-VHE. La reevaluación de anticuerpos IgG para VHE se realizó con la prueba ELISA AccuDiag ${ }^{\mathrm{TM}} \mathrm{HEV}-\mathrm{IgG}$ (Diagnostic Automation, E.U.A), el cual reporta una sensibilidad y especificidad del $99,8 \%$.

Se calculó la seroprevalencia de anticuerpos IgG para VHE para los dos ensayos diagnósticos, utilizando el test de McNemar para evaluar las diferencias significativas entre ambos, con un análisis posterior por edad y género para cada prueba mediante el test de chi cuadrado $\left(\chi^{2}\right)$.

\section{Resultados}

Se estudiaron un total de 178 muestras de sueros, correspondientes a 90 mujeres y 88 varones, la edad promedio fue de 40 años (rango: 2-86 años). Hubo 58 muestras positivas para IgG anti VHE, correspondiente a $32,6 \%$; resultado significativamente mayor al resultado previo de 


\begin{tabular}{|c|c|c|c|c|}
\hline Ensayo anti-VHE & & $\begin{array}{c}\text { Genelabs positivo } \\
n(\%)\end{array}$ & $\begin{array}{c}\text { Genelabs negativo } \\
\mathrm{n}(\%)\end{array}$ & $\begin{array}{l}\text { Total } \\
\text { n (\%) }\end{array}$ \\
\hline AccuDiag positivo & $n(\%)$ & $8(4,5)$ & $50(28,1)$ & $58(32,6)$ \\
\hline AccuDiag negativo & $n(\%)$ & $5(2,8)$ & $115(64,6)$ & $120(67,4)$ \\
\hline Total & $n(\%)$ & $13(7,3)$ & $165(92,7)$ & $178(100)$ \\
\hline
\end{tabular}

Tabla 2. Seropositividad de IgG anti VHE según grupo etario

\begin{tabular}{|cccccc|}
\hline & $\begin{array}{c}\text { Total } \\
\text { (n) }\end{array}$ & $\begin{array}{c}\text { Seropositividad } \\
\text { AccuDiag } \\
\mathbf{n}(\%)\end{array}$ & $\begin{array}{c}\text { valor } \mathbf{p} \text { (\%)V-IgG } \\
\left(\chi^{2}\right)\end{array}$ & $\begin{array}{c}\text { Seropositividad } \\
\text { Genelabs } \\
\mathbf{n}(\%)\end{array}$ & valor $\mathbf{p}$ \\
$\begin{array}{l}\text { Grupo etario } \\
\text { (años) }\end{array}$ & & & $<0,001$ & & 0,27 \\
$<20$ & 24 & $1(4,2)$ & $2(8,3)$ & \\
\hline $20-29$ & 27 & $2(7,4)$ & $0(0)$ & \\
\hline $30-39$ & 42 & $11(26,2)$ & $3(7,1)$ & \\
\hline $40-49$ & 32 & $11(34,4)$ & $2(6,3)$ & \\
\hline $50-59$ & 27 & $14(51,9)$ & & $5(18,5)$ & \\
$>59$ & 26 & $19(73,1)$ & & $1(3,8)$ & \\
\hline
\end{tabular}

$7,3 \%$ con Genelabs ( $p<0,001$ en test de McNemar) (Tabla 1). Nuestros resultados muestran un incremento de 4,5 veces en la seroprevalencia de anticuerpos IgG anti VHE al utilizar la prueba AccuDiag ${ }^{\mathrm{TM}}$ HEV-IgG. No hubo diferencias significativas en cuanto a género en los resultados de ninguna de las dos pruebas. Con el nuevo ensayo, se encontró una asociación significativa $\left(\mathrm{p}<0,001\right.$ en $\left.\chi^{2}\right)$ entre la edad y la seropositividad para IgG de VHE (Tabla 2).

\section{Discusión}

Los resultados obtenidos muestran un incremento significativo en la seroprevalencia de VHE basado en el uso de un ensayo con mejor rendimiento analítico. Existe evidencia internacional que muestra resultados similares a los nuestros, mostrando una sensibilidad variable (50-90\%) del ensayo Genelabs, que hasta hace algún tiempo era ampliamente utilizado en el mundo para los estudios de seroprevalencia ${ }^{1}$.

En un estudio reciente, Bendall y cols. compararon dos ensayos comerciales en el Reino Unido (Genelabs y Wantai) obteniendo resultados muy dispares entre ambos. La sensibilidad reportada para la detección de IgG anti-VHE fue de $56 \%$ y $98 \%$, respectivamente. Por otra parte, la seroprevalencia estimada en 500 donantes de sangre fue de 3,6\% con Genelabs y $16,2 \%$ con Wantai ${ }^{8}$. Un estudio coreano que también comparó ambas pruebas, realizado en 484 sujetos sanos, encontró una seroprevalencia de $\operatorname{IgG}$ anti-VHE de $23,1 \%$ con el ensayo Wantai y $14,3 \%$ con Genelabs ${ }^{1}$.
Wenzel y cols., compararon el desempeño analítico de tres ensayos comerciales en Alemania: MP HEV IgG ELISA (desarrollado por Genelabs, Singapur), Axiom HEV IgG EIA y Mikrogen recomLine HEV IgG, utilizando muestras de 200 sujetos sanos y encontraron seroprevalencias de IgG anti-VHE de $4,5 \% ; 29,5 \%$ y $18 \%$, respectivamente ${ }^{4}$.

Con respecto al aumento de la seroprevalencia con la edad, múltiples estudios han correlacionado la edad con mayores tasas de seroprevalencia de IgG anti-VHE, probablemente asociado a la persistencia de los anticuerpos ${ }^{1,9}$. Los títulos de IgG anti-VHE se mantienen altos hasta 4,5 años después de la infección aguda y así es como un estudio demuestra que aproximadamente en $47 \%$ de los individuos se puede detectar anticuerpos IgG anti-VHE después de 14 años posterior a la infección, lo que puede dar cuenta de las altas tasas de seroprevalencia en sujetos de mayor edad ${ }^{1}$. Es importante mencionar que los ensayos de menor sensibilidad no eran capaces de detectar títulos bajos de anticuerpos, por lo que no se evidenciaba tal correlación.

Tal como diversos estudios lo plantean, el uso de ensayos con alta sensibilidad aumenta de manera importante la seroprevalencia de IgG anti-VHE. Esto sugiere que los estudios realizados con ensayos de menor sensibilidad habrían estado subestimados y sería necesario reevaluar la seroprevalencia nacional de VHE utilizando los nuevos ensayos disponibles. De todas maneras, es necesario estandarizar las pruebas serológicas para detectar anticuerpos anti-VHE y de esta forma poder comparar los estudios realizados en las distintas poblaciones sin la influencia y variabilidad del ensayo utilizado.

Este es el primer estudio en Latinoamérica que realiza una reevaluación de la seroprevalencia de VHE utilizando los nuevos ensayos disponibles. Esta información tiene valor desde el punto de vista epidemiológico, ya que podríamos estar subestimando la importancia del VHE en nuestra población. En un futuro, se deberían actualizar los datos existentes de seroprevalencia de VHE en los bancos de sangre y en la población general utilizando los nuevos ensayos disponibles.

\section{Resumen}

En los países desarrollados se reportan seroprevalencias de virus hepatitis E (VHE) entre 0,3 y 53\%. Los datos publicados están influenciados por las cualidades técnicas de los ensayos utilizados. La sensibilidad de las nuevas pruebas ha mejorado, lo que está cambiando la seroprevalencia del VHE en el mundo.

En el presente estudio, reevaluamos en forma retrospectiva 178 muestras de suero de pacientes con estudio previo de anticuerpos IgG anti-VHE entre los años 2009 y 2012. El análisis previo se realizó con ELISA Genelabs (Singapur), siendo 7,3\% positivas. La reevaluación fue realizada con ELISA AccuDiag ${ }^{\mathrm{TM}} \mathrm{HEV}-\mathrm{IgG}$ (Diagnostic Automation, E.U.A), el cual reporta una sensibilidad y especificidad de $99,8 \%$.

Con este nuevo ensayo, encontramos $32,6 \%$ de muestras positivas, resultado significativamente mayor al resultado previo $(\mathrm{p}<0,001)$ (incremento de 4,5 veces). No hubo diferencias en género y se encontró una asociación significativa entre la edad y la seropositividad para IgG anti-VHE $(\mathrm{p}<0,001)$.

Estos resultados sugieren que los estudios previos pueden haber subestimado la seroprevalencia de VHE en Chile, la que debe ser reevaluada con los nuevos ensayos disponibles. 


\section{Referencias bibliográficas}

1.- Park H K, Jeong S H, Kim J W, Woo B H, Lee D H, Kim H Y, et al. Seroprevalence of anti-hepatitis E virus (HEV) in a Korean population: comparison of two commercial anti-HEV assays. BMC Infect Dis 2012; 12: 142 .

2.- Echevarría J M, González J E, Lewis-Ximenez L L, Dos Santos D R, Munné M S, Pinto M A, et al. Hepatitis E virus infection in Latin America: a review. J Med Virol 2013; 85: 1037-45.

3.- Teshale E H, Hu D J. Hepatitis E. Epidemiology and prevention. World J Hepatol 2011; 3: 285-91.

4.- Wenzel J J, Preiss J, Schemmerer M, Huber B, Jilg W. Test performance characteristics of Anti-HEV IgG assays strongly influence hepatitis E seroprevalence estimates. J Infect Dis 2013; 207: 497-500.
5.- Brahm J, Hurtado C, Moraga M, Gil L C, Velasco M, Alegría S, et al. Hepatitis E virus infection in Chile: preliminary report. Rev Med Chile 1996; 124: 947-9.

6.- Ibarra H, Riedemann S, Reinhardt G, Frieck P, Siegel F, Toledo C, et al. Prevalence of hepatitis E virus antibody in blood donors and other population groups in Southern Chile. Rev Med Chile 1997; 125: 275-8.

7.- Kamar N, Bendall R, Legrand-Abravanel F, Xia N, Liaz S, Isopet J, et al. Hepatitis E. Lancet 2012; 379: 2477-88.

8.- Bendall R, Ellis V, Ijaz S, Ali R, Dalton H. A comparison of two commercially available anti-HEV IgG kits and a re-evaluation of anti-HEV IgG seroprevalence data in developed countries. J Med Virol 2010; 82: 799-805.

9.- Ren F, Zhao C, Wang L, Wang Z, Gong X, Song M, et al. Hepatitis E virus seroprevalence and molecular study among blood donors in China. Transfusion 2014; 54: 910-7.

\section{Fe de Erratas}

En la carta al editor: Adherencia a la TARV en VIH: la importancia del contexto socio-cultural implicado de los autores José M. Manríquez y Valeria Stuardo, publicado en Rev Chilena Infectol 2015; 32 (3): 361-2 debe corregirse la siguiente frase de la primera columna, segundo párrafo:
Dice: En efecto, la probabilidad de mantenerse en TARV inicial, al cabo de tres años, es de 57,5\%, el 20,6\% ya está en su segunda TARV al cabo de un año es de $83 \%$.

Debe decir: En efecto, la probabilidad de mantenerse con la TARV inicial, al cabo de tres años, es de 57,5\%; el $20,6 \%$ ya está en su segunda TARV. 